\title{
The Role of Displacing Confined Solvent in the Conformational Equilibrium of $\beta$-Cyclodextrin
}

\author{
Peng $\mathrm{He}^{1, \dagger}$, Sheila Sarkar ${ }^{2}$, Emilio Gallicchio ${ }^{3}$, Tom Kurtzman ${ }^{4,5}$, \\ Lauren Wickstrom ${ }^{2, *}$
}

${ }^{1}$ Center for Biophysics \& Computational Biology/ICMS, Department of Chemistry, Temple University, Philadelphia, Pennsylvania 19122.

${ }^{2}$ Borough of Manhattan Community College, The City University of New York, Department of Science, New York, New York 10007.

${ }^{3}$ Department of Chemistry, Brooklyn College, The City University of New York, Brooklyn, New York 11210.

${ }^{4}$ Department of Chemistry, Lehman College, The City University of New York, Bronx, New York 10468.

${ }^{5}$ Ph.D. Programs in Chemistry \& Biochemistry, The Graduate Center of the City University of New York, 365 5th Avenue, New York, NY, USA 10016.

$\dagger$ Present Address: Department of Chemistry, The University of Chicago, Chicago, Illinois 60637, USA.

*Corresponding author. email: lwickstrom@ @mcc.cuny.edu, phone: 1-(212)-220-8000, ext. 7993 


\begin{abstract}
This study investigates the role of hydration and its relationship to the conformational equilibrium of the host molecule $\beta$-cyclodextrin. Molecular dynamics simulations indicate that the unbound $\beta$ cyclodextrin exhibits two state behavior in explicit solvent due to the opening and closing of its cavity. In implicit solvent, these transitions are not observed and there is one dominant conformation of $\beta$-cyclodextrin with an open cavity. Based on these observations, we investigate the hypothesis that the expulsion of thermodynamically unfavorable water molecules into the bulk plays an important role in controlling the accessibility of the closed macrostate at room temperature. We compare the results of the molecular mechanics analytical generalized Born plus non-polar solvation approach to those obtained through Grid Inhomogeneous Solvation Theory analysis with explicit solvation to elucidate the thermodynamic forces at play. The calculations help to illustrate the deficiencies of continuum solvent models and demonstrate the key role of the thermodynamics of enclosed hydration in driving the conformational equilibrium of molecules in solution.
\end{abstract}




\section{Introduction}

It is widely believed that the accurate treatment of solvation is necessary for investigating folding and binding phenomena and for the design of new drug molecules. ${ }^{1-9}$ Hydration drives and mediates dynamic biological processes through desolvation, water displacement, water reorganization, and the formation of bridging water molecules between a receptor and a ligand. Explicit and continuum molecular theories of solvation attempt to capture these effects. Implicit solvation models such as Poisson Boltzmann (PB) and generalized Born (GB) treat the solvent as a continuous medium characterized by homogeneous bulk properties and estimate the solvation free energy of a solute using specific functional forms dependent only on the solute degrees of freedom, thereby reducing the computational expense of a free energy calculation relative to an all-atom solvent description which requires extensive sampling. In contrast, the more computationally intensive explicit solvation models provide molecular details that are relevant to capturing non-linear effects and phenomena related to the discrete nature of water molecules. ${ }^{10-15}$ Such effects include structural water molecules enclosed in ligand binding cavities of receptors that need be accounted for in macromolecular models of binding. ${ }^{14,16-20}$ Thus, the comparison between explicit and implicit solvent descriptions can be a useful paradigm to discriminate continuum solvation phenomena from those that depend critically on the molecularity of water.

Computational methods that evaluate solvation thermodynamic properties are valuable for determining the role of the structure and displacement of specific water molecules. ${ }^{16,21-30}$ One such methodology, Grid Inhomogeneous Solvation Theory (GIST), maps out the solvent density and thermodynamic parameters of solvation on a grid around a solute of interest. GIST has been used to analyze the solvation of small molecules, DNA, host-guest, and protein-ligand systems and has also been incorporated into docking scoring functions. ${ }^{14,16,31-37}$ These studies have emphasized the impact of displacing high energy water molecules from the receptor surface upon ligand binding, which have been thought to indicate binding site "hot spots" in the context of drug design.

In this work, we investigate the role of water in the conformational equilibrium of $\beta$-cyclodextrin (CD). $\beta-\mathrm{CD}$ is a cyclic oligosaccharide composed of seven D-glucose monomers which form a hydrophilic surface and a hydrophobic cavity. Due to its simple molecular structure and the availability of accurate experimental binding affinities, $\beta-\mathrm{CD}$ is a popular host molecule for the study of molecular recognition. ${ }^{34,37-48}$ Recent work on $\beta-C D$ host-guest systems has demonstrated 
the importance of incorporating reorganization and water expulsion effects into implicit solvent binding affinity models. ${ }^{48}$ In these studies, implicit solvent hydration sites were developed to account for the thermodynamically unfavorable waters inside the cavity of the apo $\beta$-CD host, that when displaced by a ligand during the binding process result in an increased binding affinity. We validated the use of these customized hydration sites with a GIST map of the $\beta$-CD host that revealed the presence of thermodynamically unfavorable water molecules inside the cavity. ${ }^{34}$

In solution, molecules are often flexible and are made up of ensembles of significantly different conformations. In addition, each conformation is often surrounded by a unique solvation pattern that can impact the solvation energies and entropies. Indeed, recent studies have shown that even small structural changes to the solute can impact solvation thermodynamic profiles. ${ }^{49,50}$ In contrast, solvation thermodynamics analysis of receptors is often performed on one molecular conformation using strong restraint forces on the solute atoms. This approach can have significant limitations when multiple conformers exist. There is therefore a strong interest in understanding not only how solvation patterns depend on solute conformation but also how specific hydration effects, such as displacement of unfavorable water molecules, affect the conformational equilibrium in solution.

In contrast to the previous study, we investigate the conformational dynamics of the apo $\beta$-CD host in solution. In explicit solvent, $\beta-C D$ exists in two states when the cavity of the host is empty: the first is similar to the one observed for $\beta-\mathrm{CD}$ complexed with guests (open) and the second has a sugar closed inside the cavity (closed). Only the open state is observed in implicit solvent. Thermodynamic decomposition of the two states reveals that the open conformation is energetically favorable relative to the closed conformation in vacuum and in implicit solvent. Solvation thermodynamic calculations with explicit solvation reveal that the hydration inside the cavity of the $\beta-C D$ is more favorable in the closed conformation. This preliminary study demonstrates how we can use GIST and multiple conformers to understand the role of water in driving the conformational equilibrium of a biological molecule. 


\section{Methods}

\section{Unrestrained explicit solvent simulations}

The initial structure of the $\beta$-cyclodextrin ( $\beta$-CD) host was built and solvated using 1983 water molecules in a cubic box using Maestro (Schrodinger, LLC). We relaxed and thermalized the solvated $\beta$-CD system with the Desmond System Builder facility using default parameters. The production run was performed with the DESMOND molecular dynamics engine ${ }^{51}$ for $200 \mathrm{~ns}$ using the NPT ensemble at $300 \mathrm{~K}$ and 1 atmosphere pressure using Martyna-Tobias-Klein thermostat and barostat ${ }^{52}$ (using coupling constants of 1 and 2 ps, respectively) without restraints. These calculations employed the OPLS 2005 force field ${ }^{53,54}$ and the TIP3P explicit solvent model. ${ }^{55}$ The MD simulation was performed for $200 \mathrm{~ns}$ with a time step of $2 \mathrm{fs}$. Frames from the trajectory were saved every $10 \mathrm{ps}$.

\section{Unrestrained implicit solvent simulations}

We obtained structural ensembles of $\beta$-CD in implicit solvent using temperature replica exchange molecular dynamics (REMD) ${ }^{56}$ with a series of 8 replicas between 300 and $600 \mathrm{~K}$. Starting structures for each replica were minimized and thermalized at their corresponding temperatures for 100 ps using the Nose-Hoover Thermostat. ${ }^{57,58}$ Each T-REMD simulation was performed for $21 \mathrm{~ns}$ for each replica with a time step of $1 \mathrm{fs}$, and frames from each trajectory were saved every 2 ps. The first $1 \mathrm{~ns}$ was discarded to remove any initial structure bias which left 10,000 frames per REMD trajectory for analysis. We used the OPLS 2005 force field $^{53,54}$ and the AGBNP2 implicit solvent model ${ }^{15,59}$ and the IMPACT MD engine ${ }^{60}$ for these calculations. AGBNP2 includes an analytical pairwise descreening implementation of the generalized Born model for the electrostatic term $\left(G_{e l}\right)$, a non-polar hydration free energy estimator for the non-electrostatic term $\left(G_{n p}\right)$, and a first-shell hydration correction term $\left(G_{\text {hyd }}\right)$.

$\mathrm{G}_{\text {solvation }}=\mathrm{G}_{\mathrm{el}}+\mathrm{G}_{\mathrm{np}}+\mathrm{G}_{\mathrm{hyd}}$

The non-polar term contains two components: $\mathrm{G}_{\mathrm{cav}}$ and $\mathrm{G}_{\mathrm{vdw}}{ }^{61,62}$

$$
G_{n p}=G_{c a v}+G_{v d w}=\sum_{i} \gamma_{i} A_{i}+\sum_{i} \alpha_{i} \frac{a_{i}}{\left(B_{i}+R_{W}\right)^{3}}
$$


$\mathrm{G}_{\mathrm{cav}}$ accounts for the work required to make a cavity in solution and $\mathrm{G}_{\mathrm{vdw}}$ accounts for the solutesolvent dispersive van der Waals forces. In Equation 4, the cavity component is a function of the surface area of atom $i\left(A_{i}\right)$ and the surface tension parameter assigned to atom $i\left(\gamma_{i}\right)$ while the van der Waals dispersion term is expressed as a function of an adjustable atom type-dependent van der Waals dispersion parameter $\left(\alpha_{\mathrm{i}}\right)^{61}$, the Born radii of atom $i\left(B_{i}\right)$, the radius is of a water molecule $\left(\mathrm{R}_{\mathrm{w}}\right)$ and

$a_{i}=-\frac{16}{3} \pi \rho_{W} \varepsilon_{i w} \sigma_{i w}^{6}$

where $\rho_{\mathrm{w}}=0.033428 \AA^{3}$ is the number density of water at standard conditions and $\varepsilon_{\text {iw }}$ and $\sigma_{\text {iw }}$ are OPLS force field Lennard Jones parameters of the oxygen atom of TIP4P water. ${ }^{55}$

$\mathrm{G}_{\text {hyd }}$ accounts for first-shell hydration effects not accounted for by linear dielectric screening, such as hydrogen bonding with solvent and water ordering in the receptor binding site. The hydration correction term is estimated using an analytical intermolecular hydrogen bond potential described by the following expression:

$G_{h y d}=\sum_{w} S\left(p_{w}\right) h_{w}$

where $S\left(p_{w}\right)$ is a switching function, based on the fraction $p_{w}$ of solvent-occupied volume in the hydration site " $w$ " of the first solvation shell of the hydrogen bonding donor or acceptor groups of the solute, and $h_{w}$ is an empirical parameter adjusted to fit experimental hydration free energies of small molecules ${ }^{63}$ that accounts for the water-solute interactions not accounted for by the force field and solvation model. This correction parameter depends on the atom type of the solute (hydrogen bonding donor or acceptor, or non-polar hydrogen). The sign of this component determines whether the interactions formed with the solvent are potentially favorable or unfavorable, while its magnitude determines the strength of the excess interaction with water. In this work, we utilize custom hydration sites pointing towards the interior of the host to model the effects of displacing unfavorable water molecules into the bulk. The $\beta$-CD host cavity contains 14 hydration sites that were each assigned a value of $h_{w}$ equal to 0.4 ; this model is referred to as AGBNP2-hs in the main text. This methodology has been successfully applied in large scale binding free energy calculation of $\beta$-CD host-guest systems. ${ }^{34,48}$ An additional REMD simulation 
of the apo $\beta$-CD was performed without these customized hydration sites; this model is referred to as AGBNP2-nohs in the main text.

\section{Structural analysis of the $\beta$-CD MD trajectories}

\section{Definition of collective variables}

We characterized the open and closed conformational states observed in the unrestrained simulation of $\beta$-CD based on the orientation of each D-glucose monomer relative to the overall orientation of the $\beta-\mathrm{CD}$ ring. The orientation of the whole $\beta-\mathrm{CD}$ is determined by as the molecule axis $\vec{C}$

$\vec{C}=\sum_{n=1}^{7} \vec{C}_{n}$

where $\vec{C}_{n}$ is the normalized cross product of the distance vectors between two adjacent oxygen atoms of the linker region of the D-glucose monomers, $\vec{X}_{n-1}$ and $\vec{X}_{n}$ (Figure 1)

$\vec{C}_{n}=\frac{\vec{X}_{n-1} \times \vec{X}_{n}}{\left\|\vec{X}_{n-1} \times \vec{X}_{n}\right\|}$

The orientation of each D-glucose monomer is determined by a vector $\vec{G}_{n}$ connecting the $\mathrm{O} 3$ and C6 atoms (Figure 1). We define the collective variables of the conformational equilibrium as the orientation of each monomer relative to the molecular axis $\vec{C}$ quantified by the corresponding angle $\theta_{n}$,

$\theta_{n}=\arccos \left(\frac{\vec{C} \cdot \vec{G}_{n}}{\left|\vec{C} \cdot \vec{G}_{n}\right|}\right)$

and report the minimum angle sampled by all of the $7 \mathrm{D}$-glucose monomers as the flip angle,

$\theta_{F L I P}=\min \left(\left\{\theta_{n}\right\}\right)$

of a conformation of $\beta-C D$.

Open and closed macrostates were defined based on the direction cosine of the flip angle, which ranges from -1 to +1 . In the open conformation of $\beta-\mathrm{CD}$, all monomer vectors are mostly parallel to the molecule vector, resulting in a large $\cos \left(\theta_{\text {FLIP }}\right)$ (Figure 1-left panel). While in the closed conformation, one or more D-glucose monomers are rotated to occupy the central cavity, so the corresponding monomer vectors of those D-glucoses would have a relatively smaller $\cos \left(\theta_{F L I P}\right)$ (Figure 1-right panel). Structures of $\beta$-CD are classified as belonging to the closed conformational 
state when $\cos \left(\theta_{F L I P}\right)$ is smaller than -0.2 , and to the open conformational state when $\cos \left(\theta_{F L I P}\right)$ is greater than 0.3 .
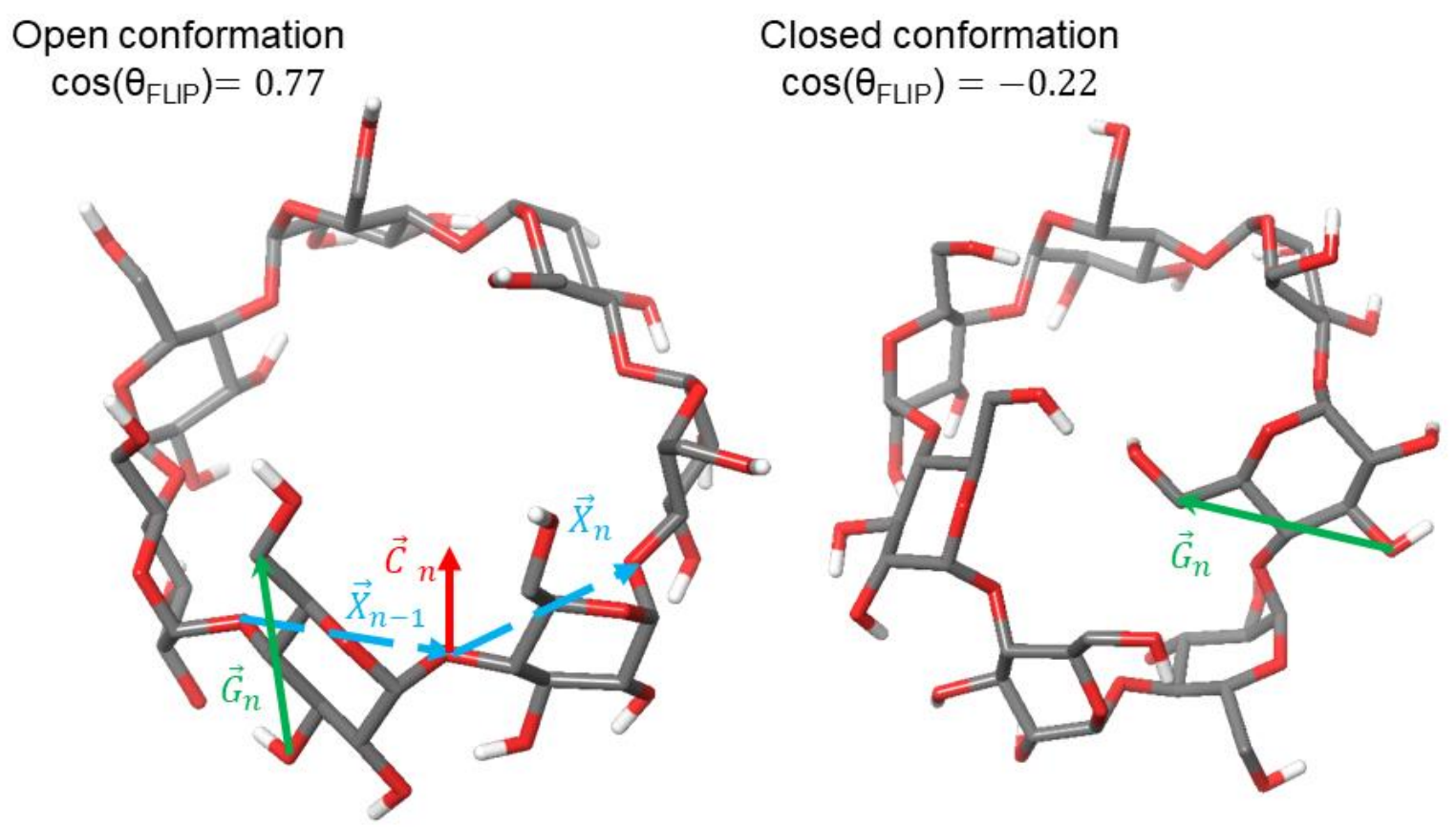

Figure 1: Definition of the collective variable used to characterize open (left panel) and closed (right panel) conformations of $\beta$-CD. The collective variable is defined as the direction cosine of the flip angle $\left(\theta_{F L I P}\right)$, the minimum angle between the molecular axis $\left(\vec{C}_{\mathrm{n}}\right)$, which is defined by distance vectors between two adjacent oxygen atoms of the linker region of the D-glucose monomers, $\vec{X}_{n-1}$ and $\vec{X}_{n}$, and the vector formed by the O3 and C6 atoms on the one of the seven D-glucose monomers $\left(\vec{G}_{n}\right)$.

Definition for counting water molecules inside the $\beta$-CD cavity in the explicit solvent simulations The transition between open and closed conformations was also characterized in terms of the number of water molecules within the interior of the host. This analysis was done to examine the relationships between solute conformation and water displacement. A water molecule is considered to be inside the $\beta$-CD cavity if its oxygen atom is located within a sphere of radius 2.8 $\AA$ centered on the center of mass of $\beta$-CD (Figure 2). 


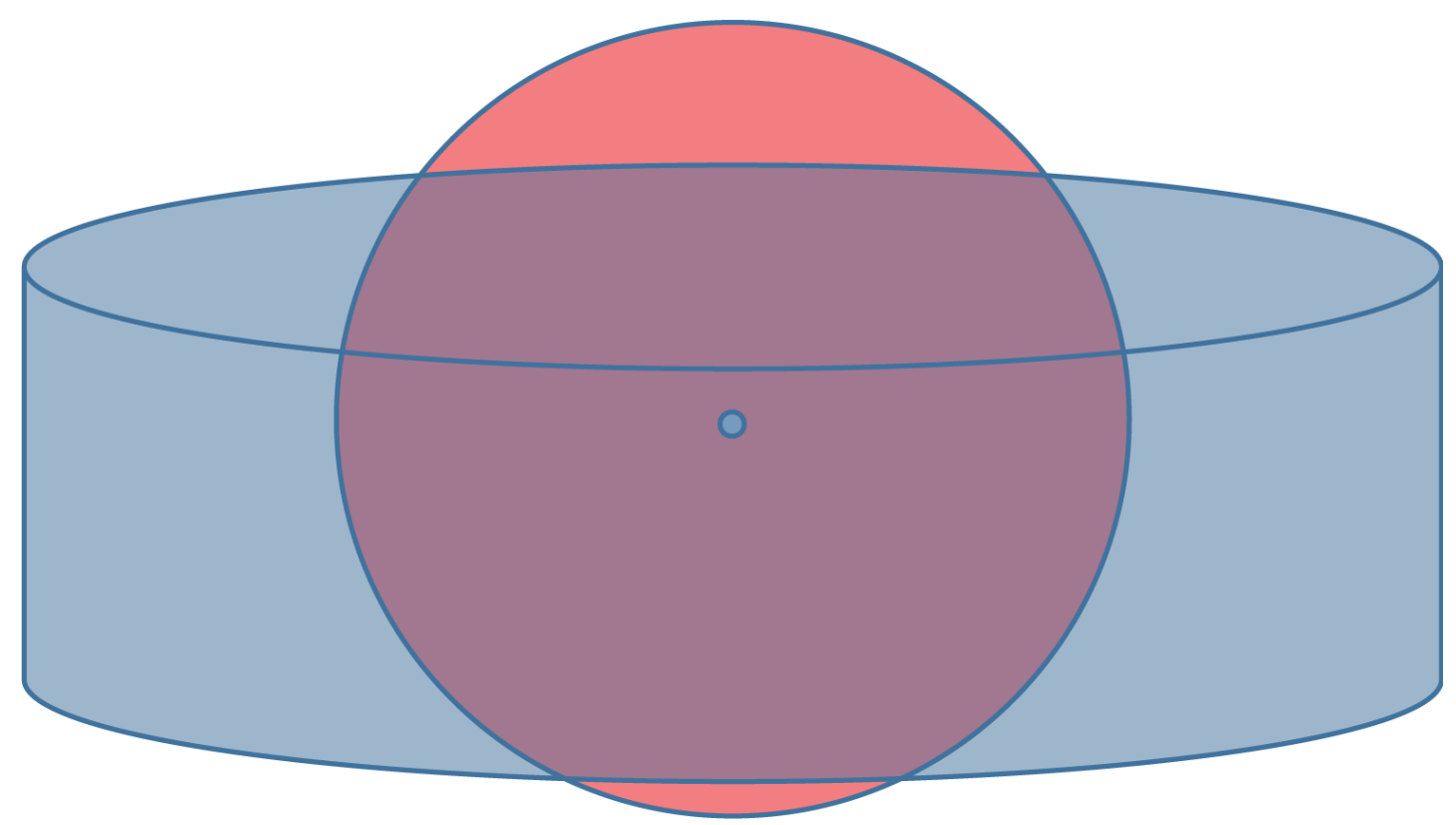

Figure 2: Illustration of the solvent region within $\beta$-CD cavity. Water molecules are considered to be inside the cavity if they are located inside the red sphere with a radius of $2.8 \AA$. The center of the sphere is the center of mass of the $\beta-C D$.

\section{Thermodynamic Analysis of the Trajectories}

$\underline{\text { Scoring using the molecular mechanics analytical generalized Born plus non-polar solvation (MM- }}$ $\underline{\text { AGBNP) and Poisson Boltzmann (PB) calculations }}$

Structures obtained from the unrestrained $\beta-C D$ simulation were postprocessed using the IMPACT software package. ${ }^{60}$ Energies were obtained for each structure using the OPLS 2005 force field ${ }^{53,54}$ in vacuo and using the AGBNP2 implicit solvent model. ${ }^{15,59}$ Poisson-Boltzmann electrostatic calculations were performed using the DelPhi program ${ }^{64}$ with a grid spacing of $0.25 \AA$. An external and internal dielectric of 80.0 and 1.0 were used to be consistent with the AGBNP2 implicit solvent model. The OPLS2005 charge and AGBNP2 radii sets were used in all of the calculations. These calculations were performed on 20,000 frames from the original unrestrained trajectory of $\beta$-CD. As discussed above, energy scores with implicit solvation were used to assess the effect of not modelling water explicitly.

\section{Grid Inhomogenous Solvation Theory (GIST)}

GIST analysis was performed on representative conformations of the open and closed conformational macrostates (Figure 1). Molecular dynamics trajectories were obtained at $300 \mathrm{~K}$ 
for $100 \mathrm{~ns}$ using the NVT ensemble with the Nose-Hoover ${ }^{57,58}$ thermostat using DESMOND ${ }^{51}$ with a time step of $2 \mathrm{fs}$. Harmonic restraints were applied to the heavy atoms of $\beta$-CD with a restrained weight of $8.0 \mathrm{kcal} / \mathrm{mol} \mathrm{A}^{2}$. Structures were written to the trajectory file every $0.5 \mathrm{ps}$. GIST was implemented on a cubic grid of dimensions $20.5 \AA$ X $20.5 \AA$ X $20.5 \AA$. GIST maps were visualized using visual molecular dynamics (vmd) software. ${ }^{65}$ 


\section{Results}

\section{Conformational dynamics of $\beta-C D$ in explicit solvent}
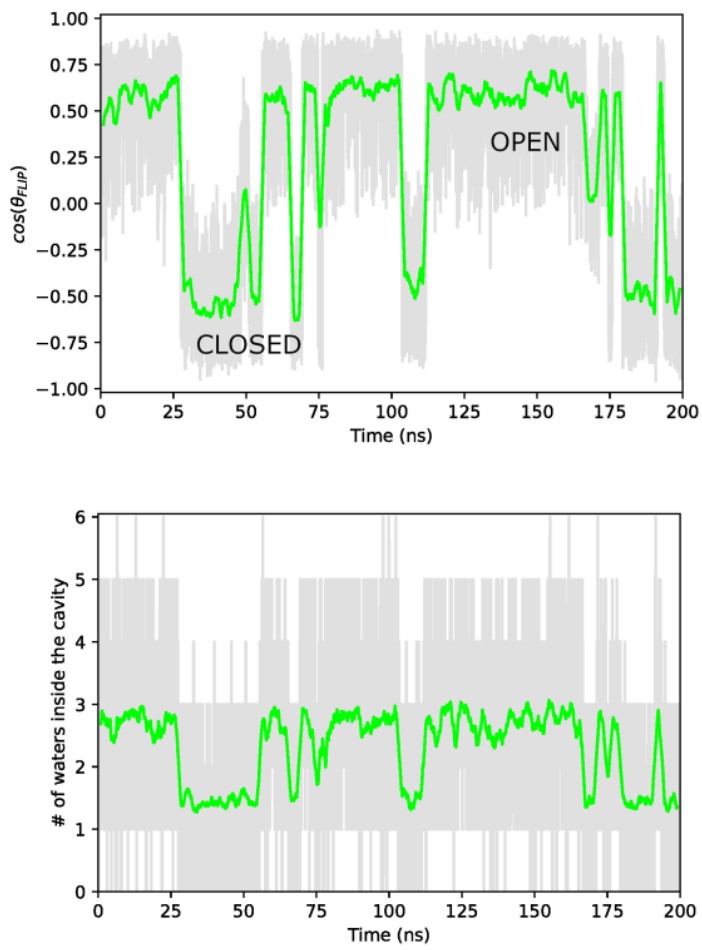

Figure 3: Time evolution of the direction cosine of the flip angle (top panel) and the number of water molecules (bottom panel) in $\beta$-cyclodextrin (gray). The running average was calculated using $2 \mathrm{~ns}$ windows (green). The open and closed states for the $\beta$-CD molecule are labeled based on their corresponding definition in the Methods section. 

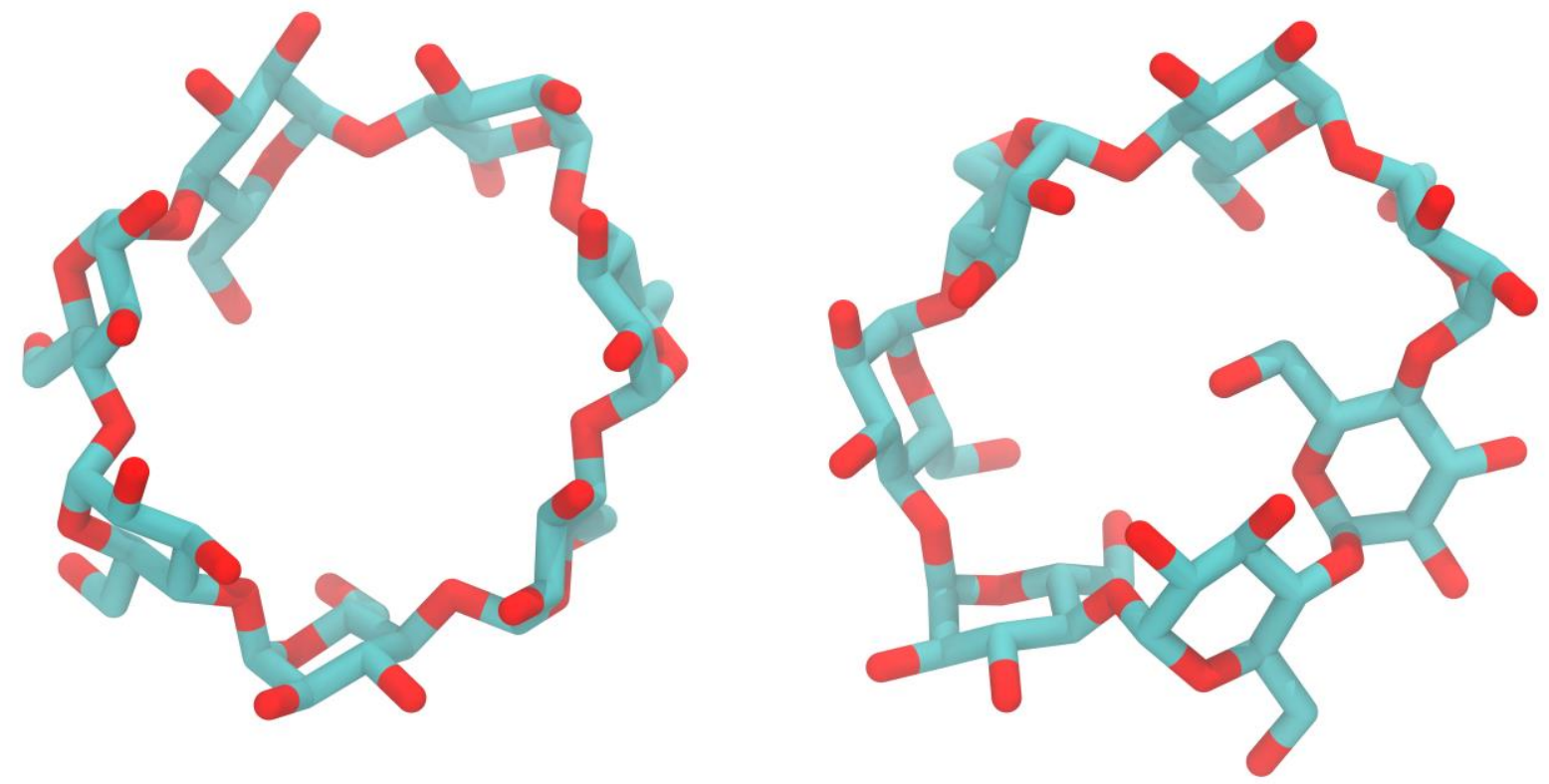

Figure 4: Conformations of $\beta$-cyclodextrin observed in the explicit solvent simulations. One conformation contained an open cavity (left panel) while the other conformation contains a monomer in the flipped conformation leaning inside the cavity (right panel).

Molecular dynamics simulations show that two states exist when the cavity of $\beta$-CD host does not contain a guest (Figure 3 ). The first state resembles the one typically observed for a $\beta$-CD-guest complex (open) and the second has a sugar monomer flipped inside the cavity (closed) (Figure 4). Initially, the simulation sampled mostly open conformations $\left(\cos \left(\theta_{F L I P}\right)>0.3\right)$ to then switch to closed conformations $\left(\cos \left(\theta_{F L I P}\right)<-0.2\right)$. These transitions continue throughout the simulations, establishing a two-state conformational equilibrium between the open and closed conformational states of $\beta-C D$. 

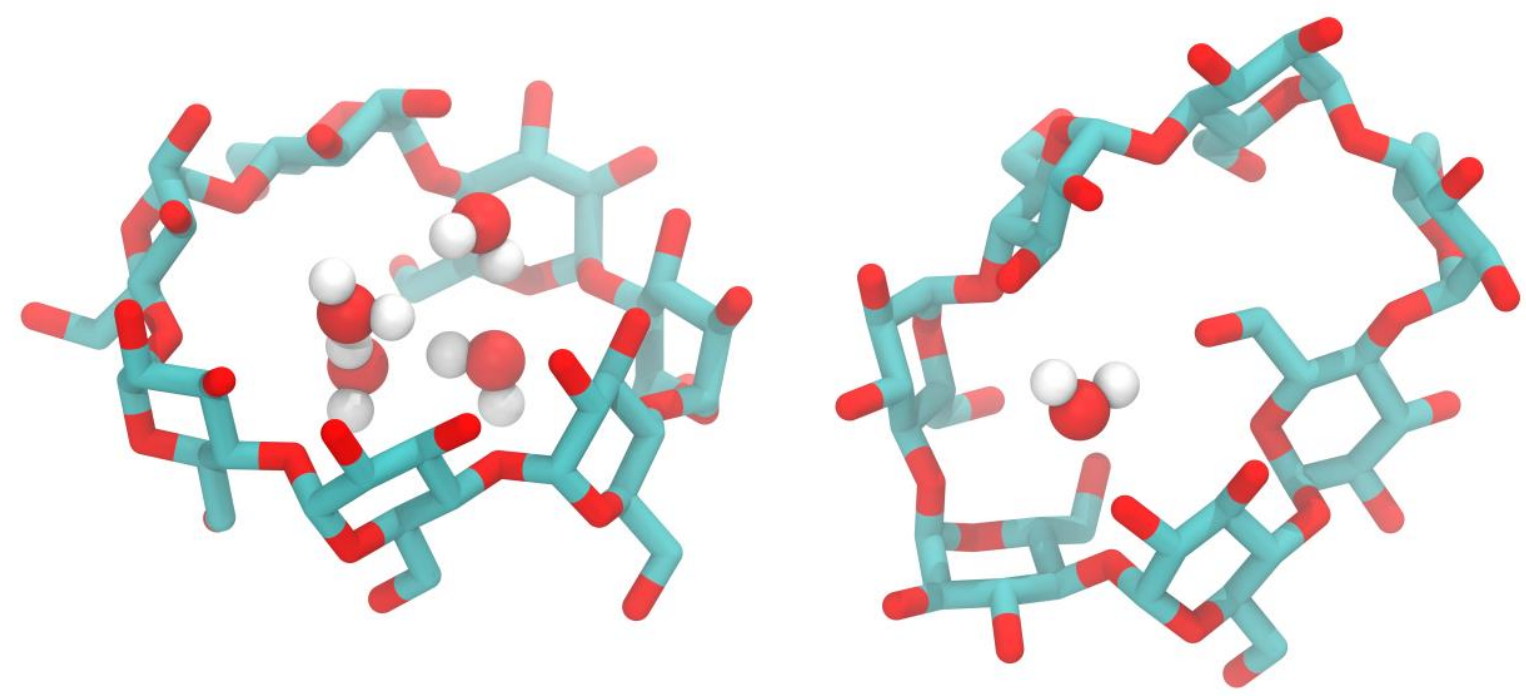

Figure 5: Representative conformations of the open (left panel) and closed states (right panel) of $\beta$-CD. Water molecules inside the host cavity are indicated in van der Waals representations. Closed conformations tend to hold fewer water molecules.

In a closed conformation, a sugar monomer partially occludes the $\beta$-CD cavity thereby reducing water occupancy (see the Methods for the definition of water occupancy). As shown in Figure 3, the $\beta$-CD cavity contains almost three water molecules on average while in the open conformation and one water molecule when in the closed conformation. It should also be noted that the range of waters is broader for the structures sampling the open conformation relative to the closed conformation (0-6 water molecules for the open vs 0-4 molecules for the closed conformation). In the open conformation, a network of water molecules is located inside the cavity and most of these water molecules are displaced in the closed conformation where a sugar monomer forms a hydrogen bond with the only water molecule, restricting its movement in and out of the cavity (Figure 5). 


\section{Comparison of Implicit and Explicit Solvent Simulation Results}

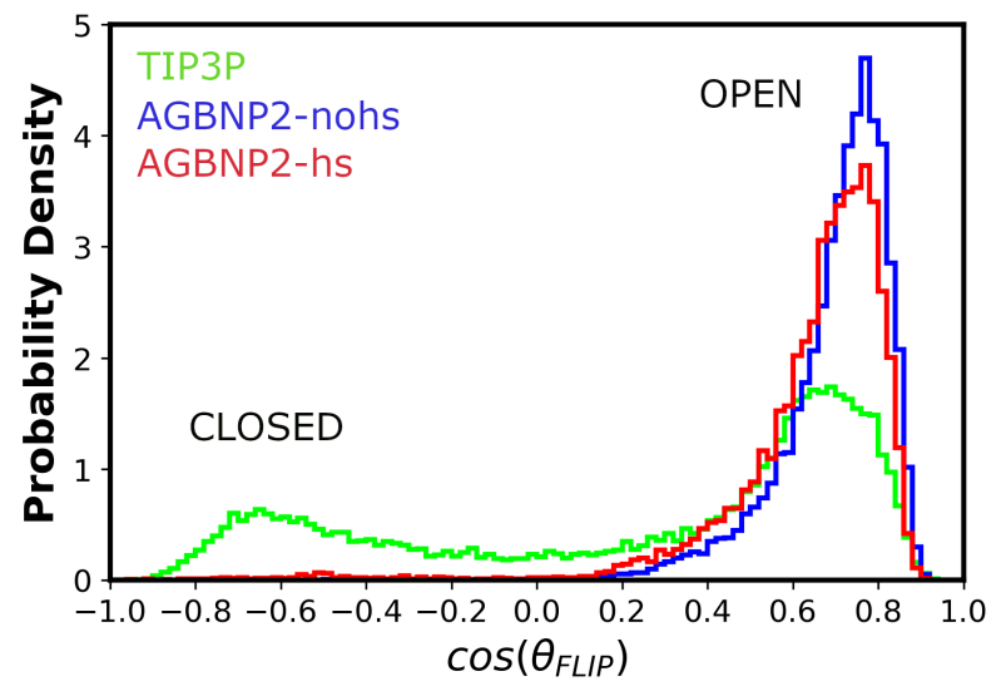

Figure 6: Histogram of the population of the direction cosine of the flip angle for the most bent sugar monomer observed in the simulations in explicit solvent (green) and implicit solvent simulations with AGBNP2 with and without custom hydration sites in the host cavity (in red and blue respectively).

To probe the role of solvation on the conformational equilibrium of $\beta-\mathrm{CD}$, we compare the populations of the open and closed states sampled in explicit and implicit solvent. The implicit solvent ensembles were generated using replica exchange molecular dynamics and the AGBNP2 implicit solvent model which incorporates the effects of solute-water hydrogen bonds as well as the free energy of water molecules confined in the host interior (see Methods). As the results in Figure 6 show, the open conformation is dominant in both explicit and implicit solvent while the closed conformation is rarely observed in implicit solvent simulations even when the unfavorable effects of confined water are considered (Figure 6). These results suggest that discrete water molecules may be necessary to sample the closed conformation in the simulation. Furthermore, by comparing the conformational distributions sampled by the AGBNP2-nohs and AGBNP2-hs models, it is apparent that the sampling of the closed conformation depends on the value of $h_{w}$ given to interior AGBNP2 hydration sites thus demonstrating the importance of enclosed water molecules in driving the equilibrium between the open and closed state. 


\section{Energetic Decomposition of Open and Closed Conformations}

Table 1: Energetic analysis of the open and closed states of $\beta$-CD observed in the explicit solvent simulation. All energies are reported in $\mathrm{kcal} / \mathrm{mol}$.

\begin{tabular}{|c|c|c|c|}
\hline & Open State & Closed State & Difference \\
\hline Population & $61.22 \%$ & $26.32 \%$ & $34.90 \%$ \\
\hline Average no. of water molecules in interior & 2.81 & 1.45 & 1.36 \\
\hline Average gas-phase potential energy & $263.2 \pm 0.2$ & $265.3 \pm 0.4$ & $-2.1 \pm 0.6$ \\
\hline Average PB hydration energy & $-110.2 \pm 0.1$ & $-109.7 \pm 0.2$ & $-0.5 \pm 0.3$ \\
\hline $\begin{array}{l}\text { Average AGBNP2 hydration energy (without } \\
\text { custom hydration interior sites) }\end{array}$ & $-66.7 \pm 0.1$ & $-66.0 \pm 0.1$ & $-0.7 \pm 0.2$ \\
\hline $\begin{array}{l}\text { Average AGBNP2 hydration energy (with custom } \\
\text { interior hydration sites) }\end{array}$ & $-62.3 \pm 0.2$ & $-63.4 \pm 0.2$ & $+1.1 \pm 0.4$ \\
\hline Average effective energy ${ }^{66}(\mathrm{~PB})$ & $153.0 \pm 0.3$ & $155.6 \pm 0.6$ & $-2.6 \pm 0.9$ \\
\hline $\begin{array}{l}\text { Average effective energy (without custom } \\
\text { hydration interior sites) }\end{array}$ & $196.5 \pm 0.3$ & $199.3 \pm 0.6$ & $-2.8 \pm 0.8$ \\
\hline $\begin{array}{l}\text { Average effective energy (with custom hydration } \\
\text { interior sites) }\end{array}$ & $200.9+/-0.4$ & $201.9+/-0.6$ & $-1+/-1$ \\
\hline
\end{tabular}

To understand the structural preferences of the $\beta-\mathrm{CD}$ host in solution, we performed energetic decomposition on the $\beta$-CD ensemble generated from the explicit solvent simulation (Table 1). The ensemble was separated into open and closed macrostates based on the direction cosine of the flip angle as defined in the Methods section. The difference in populations implies that the open state is more favorable than the closed state by only $0.5 \mathrm{kcal} / \mathrm{mol}$ of free energy. As shown in Table 1, the molecular mechanics force field (gas-phase) potential energy favors the open state by 2.1 $\mathrm{kcal} / \mathrm{mol}$ on average. The solvation contribution estimated using implicit solvation favors either the open or the closed state depending on the specific model. The Poisson Boltzmann model and the AGBNP2 model without custom hydration sites in the host interior slightly favor the open state. In contrast, the AGBNP2 model with custom hydration sites favors the closed state by approximately $1 \mathrm{kcal} / \mathrm{mol}$, thus demonstrating the influence of water displacement in favor of a 
smaller host cavity. All implicit solvent effective potential energy models (molecular mechanics + implicit solvent hydration) favor the open conformational state, particularly the Poisson Boltzmann model and the AGBNP2 model without custom hydration sites, and, to a lesser degree, the AGBNP2 model with custom hydration sites. This explains why the closed state is never sampled in the AGBNP2 simulations without custom hydration sites and is visited, albeit with small probability, with the AGBNP2 model with custom hydration sites (Figure 6).

\section{Understanding how solvation thermodynamics drive the conformational dynamics of $\beta$-CD}
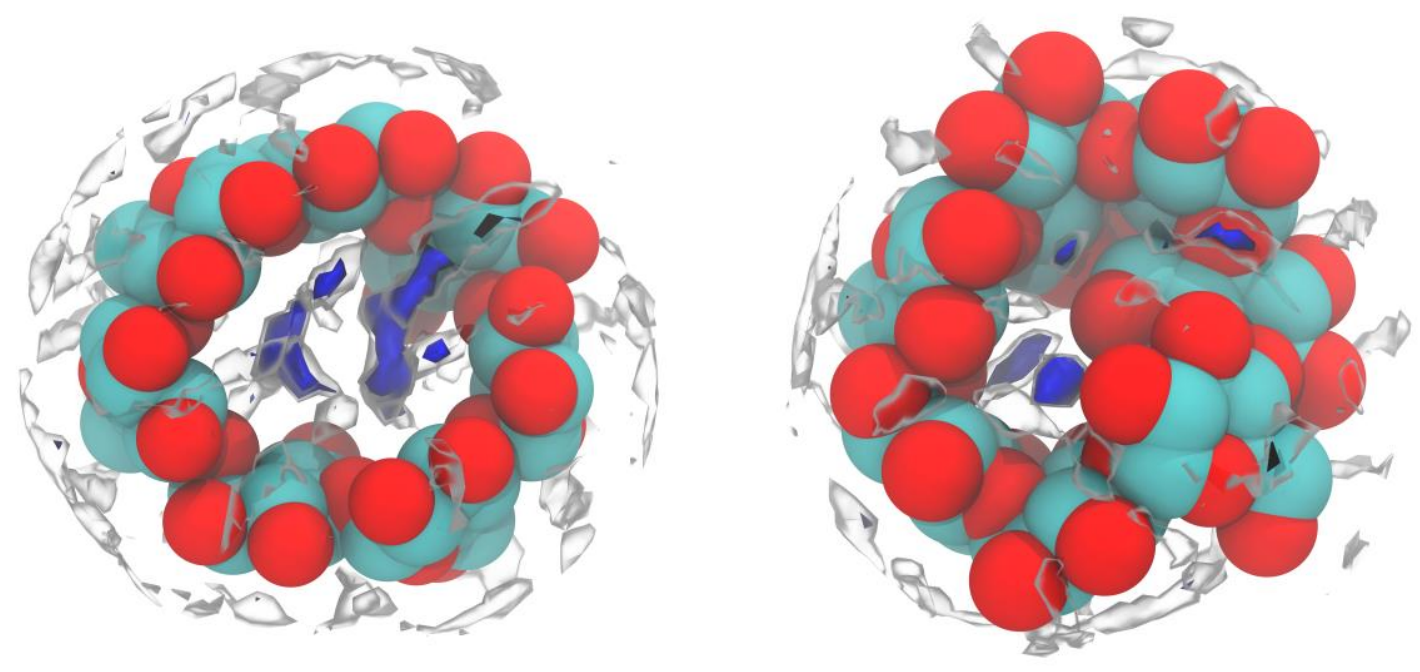

Figure 7: Contour plots of the solvation free energy for the open (left panel) and closed (right panel) conformation of $\beta$-CD for regions of water density. Contours are shown at 1.0 $\mathrm{kcal} / \mathrm{mol} /$ water (silver) and $2.0 \mathrm{kcal} / \mathrm{mol} /$ water (blue) for regions of high water density.

In order to investigate the potential effects of enclosed waters in the $\beta$-CD cavity, GIST calculations were performed on both the open and closed conformations of the apo $\beta$-CD host. Figure 7 shows the solvation free energy profiles of the high-water density regions around both conformations. The cavity of the open conformation contains a region of unfavorable hydration relative to bulk water by $2.0 \mathrm{kcal} / \mathrm{mol}$. In the closed conformation, this volume of unfavorable hydration is reduced inside the cavity due to the occupancy of the sugar. This result suggests that unfavorable solvation may play a role in the formation of the closed conformation. 
Table 2: Solvation thermodynamics inside the $\beta$-CD cavity of the open and closed conformation. Solvation thermodynamic quantities are reported in $\mathrm{kcal} / \mathrm{mol}$.

\begin{tabular}{|l|c|c|c|c|c|c|c|c|}
\hline & $\begin{array}{c}\text { Average \# } \\
\text { of waters }\end{array}$ & $\Delta \mathrm{E}_{\mathrm{sw}}$ & $\Delta \mathrm{E}_{\mathrm{ww}}$ & $\Delta \mathrm{E}_{\text {solv }}$ & $-\mathrm{T} \Delta \mathrm{S}_{\text {trans }}$ & $-\mathrm{T} \Delta \mathrm{S}_{\text {orient }}$ & $-\mathrm{T} \Delta \mathrm{S}_{\text {solv }}$ & $\Delta \mathrm{G}_{\text {solv }}$ \\
\hline Open & 3.60 & -12.11 & 15.43 & 3.32 & 1.55 & 2.31 & 3.86 & 7.18 \\
\hline Closed & 1.98 & -19.14 & 18.28 & -0.86 & 2.81 & 3.18 & 6.00 & 5.14 \\
\hline $\begin{array}{l}\text { Open- } \\
\text { Closed }\end{array}$ & 1.62 & 7.03 & -2.85 & 4.18 & -1.26 & -0.87 & -2.14 & 2.04 \\
\hline
\end{tabular}

A breakdown of the solvation thermodynamics inside the cavity of both conformations was performed to further the understanding of the role of water in this conformational switch. Table 2 shows the results of integrating the solvation thermodynamic densities inside the cavities of both conformations. The change in the free energy solvation density is approximately $2.0 \mathrm{kcal} / \mathrm{mol}$ between the open and closed conformations as 1.6 waters are displaced from the cavity of $\beta$-CD. Overall, the waters inside the open conformation are $4.1 \mathrm{kcal} / \mathrm{mol}$ energetically unfavorable relative to the closed conformation. This energetic preference for the closed state is driven by solute-water interactions that are $7.0 \mathrm{kcal} / \mathrm{mol}$ more favorable inside the $\beta$-CD host because a hydrogen bond is formed between the closed sugar and a water molecule (Figure 5). In both conformations, the water-water energies are quite unfavorable in the cavity, 15.4 and 18.2 $\mathrm{kcal} / \mathrm{mol}$, respectively, because waters inside the open cavity are unable to form the full complement of hydrogen bonds that they can in neat water. In the closed conformation, waterwater energies are extremely unfavorable because the volume of the cavity decreases, limiting potential water molecules from entering and exiting the cavity. In addition, one of the high occupancy waters inside the cavity is unable to hydrogen bond to as many neighboring water molecules since it is hydrogen bonding to the closed sugar inside the cavity (Figure 7). The effects of this bridging water are also observed in solvation entropies that are $2.0 \mathrm{kcal} / \mathrm{mol} \mathrm{more}$ unfavorable for the closed conformation than the open conformation. 


\section{Discussion}

In this work, we model the conformational equilibrium between the closed and open states of $\beta$ cyclodextrin ( $\beta-C D)$ in water solution (Figure 2). The open macrostate corresponds to the structure of holo $\beta-\mathrm{CD}$, in which the interior cavity is large enough to hold four to five water molecules. In the closed state, the host cavity is partially occluded by a glucose monomer and holds less than two water molecules on average. Previous work has also noted the existence of these two conformations in explicit solvent with different force fields and water models. ${ }^{37,45,67}$

The displacement of water from the interior of the cavity plays a critical role in the reorganization mechanism of $\beta$-CD from open to closed conformations. Water molecules enclosed in the host cavity experience unfavorable free energies relative to bulk water. It is known that displacement of unfavorable water molecules into the bulk can enhance the strength of ligands binding to receptors. ${ }^{14,16,68}$ We show that a similar mechanism is responsible for the conformational reorganization of apo $\beta-\mathrm{CD}$. When a ligand is not present a sugar monomer flips into the host cavity driven by the favorable free energy resulting from the displacement of enclosed water molecules.

Hydration-driven conformational reorganization processes such as these are significant because they can negatively affect the benefit of water displacement in ligand binding in a drug design context. To allow a ligand to enter its cavity, closed $\beta-\mathrm{CD}$ must expend free energy to reorganize into the binding-competent open conformation. Similar occurrences have been observed in the context of protein systems. In a recent joint experimental/computational study, Rechlin et al investigated the binding of a series of 2-carbamoyl-phenyloxy acetic acid derivatives to Human Aldose Reductase and found that the opening of a specific cleft in the binding pocket was dependent on ligand substituent due to protein reorganization and the ligand desolvation penalties. ${ }^{69} \mathrm{MD}$ simulations of the unbound protein found that the open conformation of this pocket was unstable relative to the closed conformation and solvation thermodynamic analysis confirmed the presence of energetically unfavorable water molecules inside the binding cleft. In another joint experimental and computational study, Maeno et al noted the existence of two states for the L99A mutant of T4 Lysozyme under high pressure conditions: a ground state with an enlarged hydrophobic cavity and a high energy state where a phenylalanine was flipped inside the cavity. ${ }^{70} 3 \mathrm{D}$-RISM analysis of the ground state X-ray conformations show that three to four 
ordered water molecules can occupy this cavity and would be displaced by the phenylalanine sidechain in the high energy state. Lastly, Oruguchi et al have noted the importance of a wetting/dewetting transition of a hydrophobic cavity for domain motion of hexameric glutamate dehydrogenase using AFM and MD simulations. ${ }^{71}$ The examples above illustrate how hydration and reorganization are intertwined and must be both considered to fully understand conformational change and binding mechanisms of biomolecules.

The thermodynamics of confined hydration is linked to the specific size and the interaction geometry of water molecules. To show that the conformational reorganization of $\beta-\mathrm{CD}$ is driven by water expulsion, we studied the process with bulk continuum dielectric models that are not expected to represent the free energy of transfer of confined water to bulk. According to our analysis, the intramolecular potential energy substantially favors the open state and so the closed state would not occur to a significant extent in the absence of hydration forces. Continuum dielectric models (AGBNP and PB) further favor the open state relative to the closed state. As a result, we do not observe formation of the closed state with these models. In contrast, the AGBNP2 hydration energies with custom hydration sites and GIST, which include water enclosure effects, favor the closed state. In explicit solvent, the solvation thermodynamics favor the closed state by $2.0 \mathrm{kcal} / \mathrm{mol}$ due to unfavorable hydration inside the cavity. This breakdown demonstrates the balance between solute energetics and solvation allowing for the sampling of both states at room temperature. These results also suggest that the molecularity and specific properties of water play a role in sampling the closed conformation.

As part of this work, we have also a developed a novel approach to calculate conformational free energy differences using MM-AGBNP-nohs to calculate the molecular mechanics energy and GIST to calculate the solvation components. Using the MM-AGBNP-GIST approach, we calculate a free energy difference of approximately $0.8 \mathrm{kcal} / \mathrm{mol}$, favoring the open state of $\beta-\mathrm{CD}$ relative to the closed state. The free energy difference based on the conformational populations in explicit solvent yields a similar free energy difference of $0.5 \mathrm{kcal} / \mathrm{mol}$. These calculated free energy differences are similar to those obtained using thermodynamic integration. ${ }^{72}$ 


\section{Conclusions}

In this work, we show that $\beta$-cyclodextrin demonstrates two-state behavior in explicit solvent. MM/AGBNP and GIST thermodynamic decomposition provides insights into how hydration inside of this host plays an important role in controlling the accessibility of these states at room temperature. Importantly, these end-state approaches using IST methodology capture important non-continuum effects that can help improve the physical nature and accuracy of implicit solvent models. Future work will focus on using the technology to understand the general mechanisms of how hydration and structural reorganization regulate molecular recognition in protein-ligand systems

\section{Author Information}

\section{Corresponding Author}

* email: 1wickstrom@bmcc.cuny.edu, phone: 1-(212)-220-8000, ext. 7993

\section{Present Addresses}

$\dagger$ Dr. Peng He is currently a postdoctoral scholar at The University of Chicago (Department of Chemistry, The University of Chicago, Chicago, Illinois 60637, USA).

\section{Author Contributions}

The manuscript was written through contributions of all authors. All authors have given approval to the final version of the manuscript.

\section{Acknowledgements}

This work was supported in part by NIH R01-GM100946 (TK). We gratefully acknowledge Amber Carr, Nanjie Deng, Michael Gilson, Ronald M. Levy, Crystal Nguyen and Steven Ramsey for providing helpful feedback for this manuscript. 


\section{References}

(1) Baron, R.; McCammon, J. A. Molecular Recognition and Ligand Association. Annu. Rev. Phys. Chem. 2013, 64 (1), 151-175. https://doi.org/10.1146/annurev-physchem-040412110047.

(2) de Beer, S. B. A.; Vermeulen, N. P. E.; Oostenbrink, C. The Role of Water Molecules in Computational Drug Design. Curr. Top. Med. Chem. 2010, 10 (1), 55-66.

(3) Hummer, G. Molecular Binding: Under Water's Influence. Nat. Chem. 2010, 2 (11), 906907. https://doi.org/10.1038/nchem.885.

(4) Li, Z.; Lazaridis, T. Water at Biomolecular Binding Interfaces. Phys. Chem. Chem. Phys. 2007, 9 (5), 573-581. https://doi.org/10.1039/B612449F.

(5) Mancera, R. L. Molecular Modeling of Hydration in Drug Design. Curr. Opin. Drug Discov. Devel. 2007, 10 (3), 275-280.

(6) Wong, S. E.; Lightstone, F. C. Accounting for Water Molecules in Drug Design. Expert Opin. Drug Discov. 2011, 6 (1), 65-74. https://doi.org/10.1517/17460441.2011.534452.

(7) Ladbury, J. E. Just Add Water! The Effect of Water on the Specificity of Protein-Ligand Binding Sites and Its Potential Application to Drug Design. Chem. Biol. 1996, 3 (12), 973-980. https://doi.org/10.1016/S1074-5521(96)90164-7.

(8) Levy, Y.; Onuchic, J. N. Water Mediation in Protein Folding and Molecular Recognition. Annu. Rev. Biophys. Biomol. Struct. 2006, 35 (1), 389-415. https://doi.org/10.1146/annurev.biophys.35.040405.102134.

(9) Ball, P. Water as an Active Constituent in Cell Biology. Chem. Rev. 2008, 108 (1), 74108. https://doi.org/10.1021/cr068037a.

(10) Haider, K.; Wickstrom, L.; Ramsey, S.; Gilson, M. K.; Kurtzman, T. Enthalpic Breakdown of Water Structure on Protein Active-Site Surfaces. J. Phys. Chem. B 2016, 120 (34), 8743-8756. https://doi.org/10.1021/acs.jpcb.6b01094.

(11) Irwin, B. W. J.; Vukovic, S.; Payne, M. C.; Huggins, D. J. Large-Scale Study of Hydration Environments through Hydration Sites. J. Phys. Chem. B 2019, 123 (19), 4220-4229. https://doi.org/10.1021/acs.jpcb.9b02490.

(12) Barelier, S.; Boyce, S. E.; Fish, I.; Fischer, M.; Goodin, D. B.; Shoichet, B. K. Roles for Ordered and Bulk Solvent in Ligand Recognition and Docking in Two Related Cavities. PLOS ONE 2013, 8 (7), e69153. https://doi.org/10.1371/journal.pone.0069153. 
(13) Onufriev, A. V.; Izadi, S. Water Models for Biomolecular Simulations. Wiley Interdiscip. Rev. Comput. Mol. Sci. 2018, 8 (2), e1347. https://doi.org/10.1002/wcms.1347.

(14) Nguyen, C. N.; Cruz, A.; Gilson, M. K.; Kurtzman, T. Thermodynamics of Water in an Enzyme Active Site: Grid-Based Hydration Analysis of Coagulation Factor Xa. J. Chem. Theory Comput. 2014, 10 (7), 2769-2780. https://doi.org/10.1021/ct401110x.

(15) Gallicchio, E.; Paris, K.; Levy, R. M. The AGBNP2 Implicit Solvation Model. J. Chem. Theory Comput. 2009, 5 (9), 2544-2564. https://doi.org/10.1021/ct900234u.

(16) Nguyen, C. N.; Young, T. K.; Gilson, M. K. Grid Inhomogeneous Solvation Theory: Hydration Structure and Thermodynamics of the Miniature Receptor Cucurbit[7]Uril. $J$. Chem. Phys. 2012, 137 (4), 044101. https://doi.org/10.1063/1.4733951.

(17) Biedermann, F.; Nau, W. M.; Schneider, H.-J. The Hydrophobic Effect RevisitedStudies with Supramolecular Complexes Imply High-Energy Water as a Noncovalent Driving Force. Angew. Chem. Int. Ed. 2014, 53 (42), 11158-11171. https://doi.org/10.1002/anie.201310958.

(18) Biela, A.; Nasief, N. N.; Betz, M.; Heine, A.; Hangauer, D.; Klebe, G. Dissecting the Hydrophobic Effect on the Molecular Level: The Role of Water, Enthalpy, and Entropy in Ligand Binding to Thermolysin. Angew. Chem. Int. Ed. 2013, 52 (6), 1822-1828. https://doi.org/10.1002/anie.201208561.

(19) Setny, P.; Baron, R.; McCammon, J. A. How Can Hydrophobic Association Be Enthalpy Driven? J. Chem. Theory Comput. 2010, 6 (9), 2866-2871. https://doi.org/10.1021/ct1003077.

(20) Barillari, C.; Taylor, J.; Viner, R.; Essex, J. W. Classification of Water Molecules in Protein Binding Sites. J. Am. Chem. Soc. 2007, 129 (9), 2577-2587. https://doi.org/10.1021/ja066980q.

(21) Sindhikara, D. J.; Hirata, F. Analysis of Biomolecular Solvation Sites by 3D-RISM Theory. J. Phys. Chem. B 2013, 117 (22), 6718-6723. https://doi.org/10.1021/jp4046116.

(22) Huggins, D. J. Application of Inhomogeneous Fluid Solvation Theory to Model the Distribution and Thermodynamics of Water Molecules around Biomolecules. Phys. Chem. Chem. Phys. 2012, 14 (43), 15106-15117. https://doi.org/10.1039/C2CP42631E. 
(23) Michel, J.; Tirado-Rives, J.; Jorgensen, W. L. Energetics of Displacing Water Molecules from Protein Binding Sites: Consequences for Ligand Optimization. J. Am. Chem. Soc. 2009, 131 (42), 15403-15411. https://doi.org/10.1021/ja906058w.

(24) Young, T.; Abel, R.; Kim, B.; Berne, B. J.; Friesner, R. A. Motifs for Molecular Recognition Exploiting Hydrophobic Enclosure in Protein-Ligand Binding. Proc. Natl. Acad. Sci. 2007, 104 (3), 808-813. https://doi.org/10.1073/pnas.0610202104.

(25) Ross, G. A.; Morris, G. M.; Biggin, P. C. Rapid and Accurate Prediction and Scoring of Water Molecules in Protein Binding Sites. PloS One 2012, 7 (3), e32036. https://doi.org/10.1371/journal.pone.0032036.

(26) Ross, G. A.; Bodnarchuk, M. S.; Essex, J. W. Water Sites, Networks, And Free Energies with Grand Canonical Monte Carlo. J. Am. Chem. Soc. 2015, 137 (47), 14930-14943. https://doi.org/10.1021/jacs.5b07940.

(27) Hu, B.; Lill, M. A. WATsite: Hydration Site Prediction Program with PyMOL Interface. $J$. Comput. Chem. 2014, 35 (16), 1255-1260. https://doi.org/10.1002/jcc.23616.

(28) Lazaridis, T. Inhomogeneous Fluid Approach to Solvation Thermodynamics. 1. Theory. $J$. Phys. Chem. B 1998, 102 (18), 3531-3541. https://doi.org/10.1021/jp9723574.

(29) Luchko, T.; Gusarov, S.; Roe, D. R.; Simmerling, C.; Case, D. A.; Tuszynski, J.; Kovalenko, A. Three-Dimensional Molecular Theory of Solvation Coupled with Molecular Dynamics in Amber. J. Chem. Theory Comput. 2010, 6 (3), 607-624. https://doi.org/10.1021/ct900460m.

(30) Ishii, Y.; Yamamoto, N.; Matubayasi, N.; Zhang, B. W.; Cui, D.; Levy, R. M. SpatiallyDecomposed Free Energy of Solvation Based on the Endpoint Density-Functional Method. J. Chem. Theory Comput. 2019, 15 (5), 2896-2912. https://doi.org/10.1021/acs.jctc.8b01309.

(31) Schauperl, M.; Czodrowski, P.; Fuchs, J. E.; Huber, R. G.; Waldner, B. J.; Podewitz, M.; Kramer, C.; Liedl, K. R. Binding Pose Flip Explained via Enthalpic and Entropic Contributions. J. Chem. Inf. Model. 2017, 57 (2), 345-354. https://doi.org/10.1021/acs.jcim.6b00483.

(32) Schauperl, M.; Podewitz, M.; Ortner, T. S.; Waibl, F.; Thoeny, A.; Loerting, T.; Liedl, K. R. Balance between Hydration Enthalpy and Entropy Is Important for Ice Binding 
Surfaces in Antifreeze Proteins. Sci. Rep. 2017, 7 (1), 11901.

https://doi.org/10.1038/s41598-017-11982-8.

(33) Nakano, M.; Tateishi-Karimata, H.; Tanaka, S.; Tama, F.; Miyashita, O.; Nakano, S.;

Sugimoto, N. Local Thermodynamics of the Water Molecules around Single- and Double-

Stranded DNA Studied by Grid Inhomogeneous Solvation Theory. Chem. Phys. Lett.

2016, 660, 250-255. https://doi.org/10.1016/j.cplett.2016.08.032.

(34) Wickstrom, L.; Deng, N.; He, P.; Mentes, A.; Nguyen, C.; Gilson, M. K.; Kurtzman, T.;

Gallicchio, E.; Levy, R. M. Parameterization of an Effective Potential for Protein-Ligand

Binding from Host-Guest Affinity Data. J. Mol. Recognit. JMR 2016, 29 (1), 10-21.

https://doi.org/10.1002/jmr.2489.

(35) Koehl, A.; Hu, H.; Maeda, S.; Zhang, Y.; Qu, Q.; Paggi, J. M.; Latorraca, N. R.; Hilger,

D.; Dawson, R.; Matile, H.; et al. Structure of the M-Opioid Receptor-G i Protein

Complex. Nature 2018, 558 (7711), 547. https://doi.org/10.1038/s41586-018-0219-7.

(36) Balius, T. E.; Fischer, M.; Stein, R. M.; Adler, T. B.; Nguyen, C. N.; Cruz, A.; Gilson, M. K.; Kurtzman, T.; Shoichet, B. K. Testing Inhomogeneous Solvation Theory in StructureBased Ligand Discovery. Proc. Natl. Acad. Sci. 2017, 114 (33), E6839-E6846. https://doi.org/10.1073/pnas.1703287114.

(37) Henriksen, N. M.; Gilson, M. K. Evaluating Force Field Performance in Thermodynamic Calculations of Cyclodextrin Host-Guest Binding: Water Models, Partial Charges, and Host Force Field Parameters. J. Chem. Theory Comput. 2017, 13 (9), 4253-4269. https://doi.org/10.1021/acs.jctc.7b00359.

(38) Del Valle, E. M. M. Cyclodextrins and Their Uses: A Review. Process Biochem. 2004, 39 (9), 1033-1046. https://doi.org/10.1016/S0032-9592(03)00258-9.

(39) Rekharsky, M. V.; Inoue, Y. Complexation Thermodynamics of Cyclodextrins. Chem. Rev. 1998, 98 (5), 1875-1918. https://doi.org/10.1021/cr970015o.

(40) Chen, W.; Chang, C.-E.; Gilson, M. K. Calculation of Cyclodextrin Binding Affinities: Energy, Entropy, and Implications for Drug Design. Biophys. J. 2004, 87 (5), 3035-3049. https://doi.org/10.1529/biophysj.104.049494.

(41) Taulier, N.; Chalikian, T. V. Hydrophobic Hydration in Cyclodextrin Complexation. J. Phys. Chem. B 2006, 110 (25), 12222-12224. https://doi.org/10.1021/jp062467n. 
(42) Szejtli, J. Introduction and General Overview of Cyclodextrin Chemistry. Chem. Rev. 1998, 98 (5), 1743-1754. https://doi.org/10.1021/cr970022c.

(43) Suárez, D.; Díaz, N. Affinity Calculations of Cyclodextrin Host-Guest Complexes: Assessment of Strengths and Weaknesses of End-Point Free Energy Methods. J. Chem. Inf. Model. 2018. https://doi.org/10.1021/acs.jcim.8b00805.

(44) Giovannelli, E.; Cioni, M.; Procacci, P.; Cardini, G.; Pagliai, M.; Volkov, V.; Chelli, R. Binding Free Energies of Host-Guest Systems by Nonequilibrium Alchemical Simulations with Constrained Dynamics: Illustrative Calculations and Numerical Validation. J. Chem. Theory Comput. 2017, 13 (12), 5887-5899.

https://doi.org/10.1021/acs.jctc.7b00595.

(45) Tang, Z.; Chang, C. A. Binding Thermodynamics and Kinetics Calculations Using Chemical Host and Guest: A Comprehensive Picture of Molecular Recognition. J. Chem. Theory Comput. 2018, 14 (1), 303-318. https://doi.org/10.1021/acs.jctc.7b00899.

(46) Zhang, H.; Yin, C.; Yan, H.; van der Spoel, D. Evaluation of Generalized Born Models for Large Scale Affinity Prediction of Cyclodextrin Host-Guest Complexes. J. Chem. Inf. Model. 2016, 56 (10), 2080-2092. https://doi.org/10.1021/acs.jcim.6b00418.

(47) Mura, P. Analytical Techniques for Characterization of Cyclodextrin Complexes in Aqueous Solution: A Review. J. Pharm. Biomed. Anal. 2014, 101, 238-250. https://doi.org/10.1016/j.jpba.2014.02.022.

(48) Wickstrom, L.; He, P.; Gallicchio, E.; Levy, R. M. Large Scale Affinity Calculations of Cyclodextrin Host-Guest Complexes: Understanding the Role of Reorganization in the Molecular Recognition Process. J. Chem. Theory Comput. 2013, 9 (7), 3136-3150. https://doi.org/10.1021/ct400003r.

(49) Yang, Y.; Lill, M. A. Dissecting the Influence of Protein Flexibility on the Location and Thermodynamic Profile of Explicit Water Molecules in Protein-Ligand Binding. J. Chem. Theory Comput. 2016, 12 (9), 4578-4592. https://doi.org/10.1021/acs.jctc.6b00411.

(50) Yang, Y.; Hu, B.; Lill, M. A. Analysis of Factors Influencing Hydration Site Prediction Based on Molecular Dynamics Simulations. J. Chem. Inf. Model. 2014, 54 (10), 29872995. https://doi.org/10.1021/ci500426q.

(51) Bowers, K. J.; Chow, E.; Xu, H.; Dror, R. O.; Eastwood, M. P.; Gregersen, B. A.; Klepeis, J. L.; Kolossvary, I.; Moraes, M. A.; Sacerdoti, F. D.; et al. Scalable Algorithms for 
Molecular Dynamics Simulations on Commodity Clusters. In Proceedings of the 2006 ACM/IEEE Conference on Supercomputing; SC '06; ACM: New York, NY, USA, 2006. https://doi.org/10.1145/1188455.1188544.

(52) Martyna, G. J.; Tobias, D. J.; Klein, M. L. Constant Pressure Molecular Dynamics Algorithms. J. Chem. Phys. 1994, 101 (5), 4177-4189. https://doi.org/10.1063/1.467468.

(53) Jorgensen, W. L.; Maxwell, D. S.; Tirado-Rives, J. Development and Testing of the OPLS All-Atom Force Field on Conformational Energetics and Properties of Organic Liquids. $J$. Am. Chem. Soc. 1996, 118 (45), 11225-11236. https://doi.org/10.1021/ja9621760.

(54) Kaminski, G. A.; Friesner, R. A.; Tirado-Rives, J.; Jorgensen, W. L. Evaluation and Reparametrization of the OPLS-AA Force Field for Proteins via Comparison with Accurate Quantum Chemical Calculations on Peptides. J. Phys. Chem. B 2001, 105 (28), 6474-6487. https://doi.org/10.1021/jp003919d.

(55) Jorgensen, W. L.; Chandrasekhar, J.; Madura, J. D.; Impey, R. W.; Klein, M. L. Comparison of Simple Potential Functions for Simulating Liquid Water. J. Chem. Phys. 1983, 79 (2), 926-935. https://doi.org/10.1063/1.445869.

(56) Sugita, Y.; Okamoto, Y. Replica-Exchange Molecular Dynamics Method for Protein Folding. Chem. Phys. Lett. 1999, 314, 141-151. https://doi.org/10.1016/S00092614(99)01123-9.

(57) Hoover, W. G. Canonical Dynamics: Equilibrium Phase-Space Distributions. Phys. Rev. A 1985, 31 (3), 1695-1697. https://doi.org/10.1103/PhysRevA.31.1695.

(58) Nosé, S. A Unified Formulation of the Constant Temperature Molecular Dynamics Methods. J. Chem. Phys. 1984, 81 (1), 511-519. https://doi.org/10.1063/1.447334.

(59) Gallicchio, E.; Levy, R. M. AGBNP: An Analytic Implicit Solvent Model Suitable for Molecular Dynamics Simulations and High-Resolution Modeling. J. Comput. Chem. 2004, 25 (4), 479-499. https://doi.org/10.1002/jcc.10400.

(60) Banks, J. L.; Beard, H. S.; Cao, Y.; Cho, A. E.; Damm, W.; Farid, R.; Felts, A. K.; Halgren, T. A.; Mainz, D. T.; Maple, J. R.; et al. Integrated Modeling Program, Applied Chemical Theory (IMPACT). J. Comput. Chem. 2005, 26 (16), 1752-1780. https://doi.org/10.1002/jcc.20292.

(61) Su, Y.; Gallicchio, E. The Non-Polar Solvent Potential of Mean Force for the Dimerization of Alanine Dipeptide: The Role of Solute-Solvent van Der Waals 
Interactions. Biophys. Chem. 2004, 109 (2), 251-260.

https://doi.org/10.1016/j.bpc.2003.11.007.

(62) Levy, R. M.; Zhang, L. Y.; Gallicchio, E.; Felts, A. K. On the Nonpolar Hydration Free Energy of Proteins: Surface Area and Continuum Solvent Models for the Solute-Solvent Interaction Energy. J. Am. Chem. Soc. 2003, 125 (31), 9523-9530. https://doi.org/10.1021/ja029833a.

(63) Gallicchio, E.; Paris, K.; Levy, R. M. The AGBNP2 Implicit Solvation Model. J. Chem. Theory Comput. 2009, 5 (9), 2544-2564. https://doi.org/10.1021/ct900234u.

(64) Li, L.; Li, C.; Sarkar, S.; Zhang, J.; Witham, S.; Zhang, Z.; Wang, L.; Smith, N.; Petukh, M.; Alexov, E. DelPhi: A Comprehensive Suite for DelPhi Software and Associated Resources. BMC Biophys. 2012, 5, 9. https://doi.org/10.1186/2046-1682-5-9.

(65) Humphrey, W.; Dalke, A.; Schulten, K. VMD: Visual Molecular Dynamics. J. Mol. Graph. 1996, 14 (1), 33-38, 27-28.

(66) Lazaridis, T.; Karplus, M. Effective Energy Functions for Protein Structure Prediction. Curr. Opin. Struct. Biol. 2000, 10 (2), 139-145.

(67) Gebhardt, J.; Kleist, C.; Jakobtorweihen, S.; Hansen, N. Validation and Comparison of Force Fields for Native Cyclodextrins in Aqueous Solution. J. Phys. Chem. B 2018, 122 (5), 1608-1626. https://doi.org/10.1021/acs.jpcb.7b11808.

(68) Pal, R. K.; Haider, K.; Kaur, D.; Flynn, W.; Xia, J.; Levy, R. M.; Taran, T.; Wickstrom, L.; Kurtzman, T.; Gallicchio, E. A Combined Treatment of Hydration and Dynamical Effects for the Modeling of Host-Guest Binding Thermodynamics: The SAMPL5 Blinded Challenge. J. Comput. Aided Mol. Des. 2017, 31 (1), 29-44. https://doi.org/10.1007/s10822-016-9956-6.

(69) Rechlin, C.; Scheer, F.; Terwesten, F.; Wulsdorf, T.; Pol, E.; Fridh, V.; Toth, P.; Diederich, W. E.; Heine, A.; Klebe, G. Price for Opening the Transient Specificity Pocket in Human Aldose Reductase upon Ligand Binding: Structural, Thermodynamic, Kinetic, and Computational Analysis. ACS Chem. Biol. 2017, 12 (5), 1397-1415. https://doi.org/10.1021/acschembio.7b00062.

(70) Maeno, A.; Sindhikara, D.; Hirata, F.; Otten, R.; Dahlquist, F. W.; Yokoyama, S.; Akasaka, K.; Mulder, F. A. A.; Kitahara, R. Cavity as a Source of Conformational Fluctuation and High-Energy State: High-Pressure NMR Study of a Cavity-Enlarged 
Mutant of T4Lysozyme. Biophys. J. 2015, 108 (1), 133-145.

https://doi.org/10.1016/j.bpj.2014.11.012.

(71) Oroguchi, T.; Nakasako, M. Changes in Hydration Structure Are Necessary for Collective Motions of a Multi-Domain Protein. Sci. Rep. 2016, 6, 26302.

https://doi.org/10.1038/srep26302.

(72) Harris, R. C.; Deng, N.; Levy, R. M.; Ishizuka, R.; Matubayasi, N. Computing Conformational Free Energy Differences in Explicit Solvent: An Efficient Thermodynamic Cycle Using an Auxiliary Potential and a Free Energy Functional Constructed from the End Points. J. Comput. Chem. 2017, 38 (15), 1198-1208. https://doi.org/10.1002/jcc.24668. 